\title{
On Quasi-Sasakian 3-Manifolds with Respect to the Schouten-van Kampen Connection
}

\author{
Selcen Yüksel Perktaş* and Ahmet Yildiz
}

(Communicated by Yusuf Yaylı)

\begin{abstract}
In this paper we study some soliton types on a quasi-Sasakian 3-manifold with respect to the Schouten-van Kampen connection.
\end{abstract}

Keywords: Quasi-Sasakian manifolds; Schouten-van Kampen connection; Ricci soliton; $\eta$-Ricci soliton; Yamabe soliton.

AMS Subject Classification (2020): Primary: 53C25 ; Secondary: 53D15.

\section{Introduction}

One of the important classes of almost contact metric manifolds is the class consisting of those which are normal. However, the curvature nature of such manifolds is not known in general, except for Sasakian or cosymplectic manifolds. If the almost contact metric structure of an almost contact metric manifold is normal and the fundamental 2-form is closed then the manifold is said to be quasi-Sasakian manifold. Quasi-Sasakian structures can be considered as unifying Sasakian and cosymplectic structures and first examples were given by Blair [4]. Some characterizations related to quasi-Sasakian manifolds were given by Tanno [34] (see also [14], $[20,21],[26])$. Kim proved that fibred Riemannian spaces endowed with invariant fibres normal to the structure vector field do not admit neither nearly Sasakian nor contact structure but such spaces admit a quasi-Sasakian or a cosymplectic structure [19]. Recently, due to the significant applications to physics, in particular to super gravity and magnetic theory, and the applications in the mathematical analysis of string theory, quasi-Sasakian manifolds have been studying intensively.

Olszak defined the structure function $\beta$ on a quasi-Sasakian 3-manifold and obtained the necessary and sufficent conditions for such manifolds to be conformally flat via structure function. Also, he proved that if the manifold is additionally conformally flat with $\beta=$ constant, then (a) the manifold is locally a product of $\mathbb{R}$ and a two-dimensional Kaehlerien space of constant Gauss curvature (the cosymplectic case), or, (b) the manifold is of constant positive curvature (the non-cosympletic case, here the quasi-Sasakian structure is homothetic to a Sasakian structure) [24].

The Schouten-van Kampen connection is one of the most natural connections adapted to a pair of complementary distributions on a differentiable manifold endowed with an affine connection [5, 16, 28]. Solov'ev has investigated hyperdistributions in Riemannian manifolds using the Schouten-van Kampen connection [30, 31, 32, 33]. Olszak studied the Schouten-van Kampen connection to adapted to an almost contact metric structure in [25]. He also characterized some classes of almost contact metric manifolds endowed with the Schouten-van Kampen connection by giving certain curvature properties of this connection on such manifolds.

As a generalization of Einstein metrics, a Riemannian metric $g$ on a Riemannian manifold is called a Ricci soliton [15] if there exists a smooth vector field $v$ on the manifold satisfying

$$
L_{v} g+2 R i c+2 \rho g=0
$$


where $L$ is the Lie derivative, Ric is the Ricci tensor, and $\rho$ is a real constant. A Ricci soliton is said to be shrinking, steady and expanding according as $\rho$ is negative, zero and positive, respectively. Compact Ricci solitons are the fixed points of the Ricci flow $\frac{\partial}{\partial t} g=-2$ Ric projected from the space of metrics onto its quotient modulo diffeomorphisms and scalings, and often arise as blow-up limits for the Ricci flow on compact manifolds. . Also Cho and Kimura introduced the notion of $\eta$-Ricci soliton [8]. A Riemannian manifold ( $M, g$ ) is called an almost $\eta$-Ricci soliton if there exist a smooth vector field $v$ such that the Ricci tensor satisfies the following equation

$$
L_{v} g+2 R i c+2 \rho g+2 \sigma \eta \otimes \eta=0
$$

where $\rho$ and $\mu$ are real constants. If the vector field $v$ is the gradient of a potential function $-k$, then $g$ is called a gradient Ricci soliton and equation (1.1) assumes the form

$$
\nabla \nabla k=R i c+\mu g
$$

In dimension 2 and in dimension 3, a Ricci soliton on a compact manifold has constant curvature [15, 17]. For details we refer to Chow and Knopf [9] and Derdzinski [12]. We also recall that a Ricci soliton on a compact manifold is a gradient Ricci soliton [27].

In order to obtain self-similar solutions for Yamabe problem which seem to be as singularity models, the notion of Yamabe flow was firstly defined by Hamilton [15]. Yamabe solitons have been studied intensively and for further reading we refer $[1,6,7,13,22]$. If there exists a vector field $v$ on a Riemannian manifold $(M, g)$ satisfying [1]

$$
\frac{1}{2}\left(L_{v} g\right)=(s c a l-\delta) g,
$$

where scal is the scalar curvature of $M, \delta$ is a real constant, $v$ is a soliton field and $L$ is the Lie-derivative, then the manifold is said to be a Yamabe soliton and we denote a Yamabe soliton by $(M, v, \delta)$. Also, a Yamabe soliton is said to be steady, expanding or shrinking, if $\delta=0, \delta<0$ or $\delta>0$, respectively. If $\delta$ is a constant, then an almost Yamabe soliton becomes a Yamabe soliton. Note that, if $(M, g)$ is of constant scalar curvature scal then the Riemannian metric $g$ is called a Yamabe metric.

This paper has been organized as follows: After preliminaries in section 3, we have introduced quasiSasakian 3-manifolds endowed with the Schouten-van Kampen connection. In section 4, we have studied Ricci solitons, $\eta$-Ricci solitons, gradient Ricci solitons and Yamabe solitons in a quasi-Sasakian 3-manifold with respect to the Schouten-van Kampen connection. In section 5, we have reconstructed examples of a quasiSasakian 3-manifold endowed with the Schouten-van Kampen connection.

\section{Preliminaries}

A connected differentiable manifold $M$ of dimension $(2 n+1)$ is called an almost contact metric manifold, if there exist tensor fields $\varphi, \xi, \eta$ on $M$ of types $(1,1),(1,0),(0,1)$, respectively, such that $[2,3,35]$

$$
\begin{gathered}
\varphi^{2}=-I+\eta \otimes \xi, \quad \eta(\xi)=1, \\
g(\varphi U, \varphi V)=g(U, V)-\eta(U) \eta(V), \quad U, V \in T M,
\end{gathered}
$$

where $T M$ is the Lie algebra of vector fields of the manifold $M$. In this case we have $\varphi \xi=0, \eta \circ \varphi=0$ and $\eta(U)=g(U, \xi)$. The fundamental 2 -form $\Phi$ of $M$ is defined by

$$
\Phi(U, V)=g(U, \varphi V) \quad U, V \in T M
$$

An almost contact metric manifold $(M, \varphi, \xi, \eta)$ is said to be quasi-Sasakian manifold [4] if the almost contact structure is normal and the fundamental 2 -form is closed, that is, for every $U, V \in \varepsilon^{(2 n+1)}$,

$$
\begin{gathered}
{[\varphi, \varphi](U, V)+d \eta(U, V) \xi=0,} \\
d \Phi=0, \quad \Phi(U, V)=g(U, \varphi V),
\end{gathered}
$$

where $\varepsilon^{(2 n+1)}$ denotes the module of vector fields on $M$. There exist different types of quasi-Sasakian structures ranging from the cosymplectic case, $d \eta=0(\operatorname{rank} \eta=1)$, to the Sasakian case, $\eta \wedge(d \eta)^{n} \neq 0($ rank $\eta=2 n+1$, $\Phi=d \eta)$. The 1 -form $\eta$ has rank $r=2 p$ if $d \eta^{p} \neq 0$ and $\eta \wedge(d \eta)^{p}=0$, and has rank $r=2 p+1$ if $d \eta^{p}=0$ and 
$\eta \wedge(d \eta)^{p} \neq 0$, where $r$ is called the rank of the quasi-Sasakian structure. Blair also proved that there is no quasi-Sasakian manifold of even rank [4].

Now we consider a quasi-Sasakian 3-manifold (i.e, a quasi-Sasakian manifold of dimension 3). An almost contact metric manifold $M$ is a quasi-Sasakian 3-manifold if and only if [23]

$$
\nabla_{U} \xi=-\beta \varphi U, \quad U \in T M,
$$

for a certain function $\beta$ on $M$, such that $\xi \beta=0$ and $\nabla$ being the operator of the covariant differentiation with respect to the Riemannian connection of $M$. Clearly, such a quasi-Sasakian manifold is cosymplectic if and only if $\beta=0$ [18]. As a consequence of (2.3), we have [23]

$$
\begin{gathered}
\left(\nabla_{U} \varphi\right) V=\beta(g(U, V) \xi-\eta(V) U), \\
\left(\nabla_{U} \eta\right) V=-\beta g(\varphi U, V) .
\end{gathered}
$$

Using (2.3) and (2.4), we find

$$
\nabla_{U}\left(\nabla_{V} \xi\right)=-U[\beta] \varphi V-\beta^{2}\{g(U, V) \xi-\eta(V) U\}-\beta \varphi \nabla_{U} V
$$

which implies that

$$
R(U, V) \xi=-U[\beta] \varphi V+V[\beta] \varphi U+\beta^{2}\{\eta(V) U-\eta(U) V\} .
$$

Moreover (2.3) and (2.4) give the following relations:

$$
\begin{gathered}
R(U, \xi) \xi=\beta^{2}\{U-\eta(U) \xi\} \\
R(U, \xi) V=-U[\beta] \varphi V-\beta^{2}\{g(U, V) \xi-\eta(V) U\} .
\end{gathered}
$$

It is well known that in a Riemannian 3-manifold, we always have

$$
\begin{aligned}
R(U, V) W= & g(V, W) Q U-g(U, W) Q V+\operatorname{Ric}(V, W) U-\operatorname{Ric}(U, W) V \\
& -\frac{s c a l}{2}\{g(V, W) U-g(U, W) V\},
\end{aligned}
$$

where $Q$ is the Ricci operator, that is, $\operatorname{Ric}(U, V)=g(Q U, V)$ and scal is the scalar curvature of the manifold.

The Ricci tensor Ric of a quasi-Sasakian 3-manifold $M$ is given by

$$
\begin{aligned}
\operatorname{Ric}(V, W)= & \left(\frac{s c a l}{2}-\beta^{2}\right) g(V, W)+\left(3 \beta^{2}-\frac{s c a l}{2}\right) \eta(V) \eta(W) \\
& -\eta(V) d \beta(\varphi W)-\eta(W) d \beta(\varphi V),
\end{aligned}
$$

where $s c a l$ is the scalar curvature of $M$. As a consequence of (2.10), for the Ricci operator $Q$, one can write

$$
\begin{aligned}
Q U= & \left(\frac{s c a l}{2}-\beta^{2}\right) U+\left(3 \beta^{2}-\frac{s c a l}{2}\right) \eta(U) \xi \\
& +\eta(U)(\varphi \operatorname{grad} \beta)-d \beta(\varphi U) \xi,
\end{aligned}
$$

where $d \beta(U)=g(\operatorname{grad} \beta, U)$. From (2.10), we also have

$$
\operatorname{Ric}(U, \xi)=2 \beta^{2} \eta(U)-d \beta(\varphi U) .
$$

On the other hand we have two naturally defined distribution in the tangent bundle $T M$ of $M$ as follows:

$$
H=\operatorname{ker} \eta, \quad \tilde{H}=\operatorname{span}\{\xi\} .
$$

Then we have $T M=H \oplus \tilde{H}, H \cap \tilde{H}=\{0\}$ and $H \perp \tilde{H}$. This decomposition allows one to define the Schoutenvan Kampen connection $\stackrel{\star}{\nabla}$ over an almost contact metric structure. The Schouten-van Kampen connection $\stackrel{\star}{\nabla}$ on an almost contact metric manifold with respect to Levi-Civita connection $\nabla$ is defined by [30]

$$
\stackrel{\star}{\nabla}_{U} V=\nabla_{U} V-\eta(V) \nabla_{U} \xi+\left(\nabla_{U} \eta\right)(V) \xi .
$$

Thus with the help of the Schouten-van Kampen connection (2.13), many properties of some geometric objects connected with the distributions $H, V$ can be characterized [30,31,32]. For example $g, \xi$ and $\eta$ are parallel with respect to $\stackrel{\star}{\nabla}$, that is, $\stackrel{\star}{\nabla} \xi=0, \stackrel{\star}{\nabla} g=0, \stackrel{\star}{\nabla} \eta=0$. Also the torsion $\stackrel{\star}{T}$ of $\stackrel{\star}{\nabla}$ is defined by

$$
\stackrel{\star}{T}(U, V)=\eta(U) \nabla_{V} \xi-\eta(V) \nabla_{U} \xi+2 d \eta(U, V) \xi .
$$




\section{Quasi-Sasakian 3-manifolds with respect to the Schouten-van Kampen connection}

In a quasi-Sasakian 3-manifold using (2.4) and (2.5) in (2.13), we have

$$
\stackrel{\star}{\nabla}_{U} V=\nabla_{U} V+\beta \eta(V) \varphi U+\beta g(U, \varphi V) \xi .
$$

Let $R$ and $\stackrel{\star}{R}$ be the curvature tensors of the Levi-Civita connection $\nabla$ and the Schouten-van Kampen connection $\stackrel{\star}{\nabla}$

$$
R(U, V)=\left[\nabla_{U}, \nabla_{V}\right]-\nabla_{[U, V]}, \quad \stackrel{\star}{R}(U, V)=\left[\stackrel{\star}{\nabla}_{U}, \stackrel{\star}{\nabla}_{V}\right]-\stackrel{\star}{\nabla}_{[U, V]}
$$

If we substitute equation (2.13) in the definition of the Riemannian curvature tensor

$$
\stackrel{\star}{R}(U, V) W=\stackrel{\star}{\nabla}_{U} \stackrel{\star}{\nabla}_{V} W-\stackrel{\star}{\nabla}_{V} \stackrel{\star}{\nabla}_{U} W-\stackrel{\star}{\nabla}_{[U, V]} W .
$$

Using (3.1) in (3.2), we have

$$
\begin{aligned}
\stackrel{\star}{R}(U, V) W= & \stackrel{\star}{\nabla}_{U}\left(\nabla_{V} W+\beta \eta(W) \varphi V+\beta g(V, \varphi W) \xi\right) \\
& -{ }^{\star}{ }_{V}\left(\nabla_{U} W+\beta \eta(W) \varphi U+\beta g(U, \varphi W) \xi\right) \\
& -\left(\nabla_{[U, V]} W+\beta \eta(W) \varphi[U, V]+\beta g([U, V], \varphi W) \xi .\right.
\end{aligned}
$$

Using (2.4) and (2.5) in (3.3), we obtain the following formula connecting $R$ and $\stackrel{\star}{R}$ on $M$

$$
\begin{aligned}
\stackrel{\star}{R}(U, V) W= & R(U, V) W+U[\beta]\{g(V, \varphi W) \xi+\eta(W) \varphi V\} \\
& -V[\beta]\{g(U, \varphi W) \xi+\eta(W) \varphi U\} \\
& +\beta^{2}\{g(U, W) \eta(V) \xi-g(V, W) \eta(U) \xi+\eta(U) \eta(W) V \\
& -\eta(V) \eta(W) U+g(U, \varphi W) \varphi V-g(V, \varphi W) \varphi U\} .
\end{aligned}
$$

Now taking the inner product in (3.4) with a vector field $W$, we have

$$
\begin{aligned}
g(\stackrel{\star}{R}(U, V) W, Z)= & g(R(U, V) W, Z) \\
& +U[\beta]\{g(V, \varphi W) \eta(Z)+g(\varphi V, Z) \eta(W)\} \\
& -V[\beta]\{g(U, \varphi W) \eta(Z)+g(\varphi U, Z) \eta(W)\} \\
& +\beta^{2}\{g(U, W) \eta(V) \eta(Z)-g(V, W) \eta(U) \eta(Z) \\
& +g(V, Z) \eta(U) \eta(W)-g(U, Z) \eta(V) \eta(W) \\
& +g(U, \varphi W) g(\varphi V, Z)-g(V, \varphi W) g(\varphi U, Z)\} .
\end{aligned}
$$

If we do summation over $i$ by choosing $U=Z=e_{i}$ in (3.5), $\{i=1,2,3\}$, where $e_{i}$ is an orthonormal basis of the tangent space at each point of the manifold, we get

$$
\stackrel{\star}{\operatorname{Ric}}(V, W)=\operatorname{Ric}(V, W)+(\varphi V)[\beta] \eta(W)-2 \beta^{2} \eta(V) \eta(W),
$$

where $\stackrel{\star}{\star} i c$ and Ric denote the Ricci tensor of the connections $\stackrel{\star}{\nabla}$ and $\nabla$, respectively. As a consequence of (3.6), we get for the Ricci operator $\stackrel{\star}{Q}$

$$
\stackrel{\star}{Q} V=Q V+(\varphi V)[\beta] \xi-2 \beta^{2} \eta(V) \xi .
$$

Also if we take summation over $i$, where $V=W=e_{i}$ in (3.6), we get

$$
\stackrel{\star}{\star s a l}=\operatorname{scal}-2 \beta^{2},
$$

where $\stackrel{\star}{\text { scal }}$ and scal denote the scalar curvatures of the connections $\stackrel{\star}{\nabla}$ and $\nabla$, respectively. 


\section{Soliton types on quasi-Sasakian 3-manifolds with respect to the Schouten-van Kampen connection}

In this section we study Ricci solitons, $\eta$-Ricci solitons and Yamabe solitons on a quasi-Sasakian 3-manifolds with respect to the Schouten-van Kampen connection. In a quasi-Sasakian 3-manifold endowed with the Schouten-van Kampen connection, since $\stackrel{\star}{\nabla} g=0$ and $\stackrel{\star}{T} \neq 0$, by using (3.1), we get

$$
\left(\stackrel{\star}{L}_{v} g\right)(U, V)=g\left(\nabla_{U} v, V\right)+g\left(U, \nabla_{V} v\right)=\left(L_{v} g\right)(U, V),
$$

for any $U, V \in T M$.

Now we consider a non-cosymplectic quasi-Sasakian 3-manifold $M$ bearing a Ricci soliton defined by (1.1) with respect to the Schouten-van Kampen connection. Let $v$ be a pointwise collinear vector field with the structure vector field $\xi$, that is $v=f \xi$, where $f$ is a function on $M$. From (1.1) and (4.1) we write

$$
g\left(\nabla_{U} f \xi, V\right)+g\left(U, \nabla_{V} f \xi\right)+2 \stackrel{\star R i c}{\star}(U, V)+2 \rho g(U, V)=0 .
$$

Then by using (2.3), we have

$$
U[f] \eta(V)+V[f] \eta(U)+2 \stackrel{\star}{R i c}(U, V)+2 \rho g(U, V)=0,
$$

which implies

$$
U[f] \eta(V)+V[f] \eta(U)+2 \operatorname{Ric}(U, V)+2(\varphi U)[\beta] \eta(V)-4 \beta^{2} \eta(U) \eta(V)+2 \rho g(U, V)=0,
$$

by virtue of (3.6). By putting $V=\xi$ in (4.4) and using (2.12) we obtain

$$
U[f]+(\xi f) \eta(U)+2 \rho \eta(U)=0 .
$$

Taking $U=\xi$ in (4.5) gives

$$
\xi f=-\rho
$$

If we replace (4.6) in (4.5), we get

$$
U[f]=-\rho \eta(U)
$$

which yields

$$
d f=-\rho \eta .
$$

Applying $d$ on both sides of the last equation, we have

$$
\rho d \eta=0 .
$$

In a non-cosymplectic quasi-Sasakian 3-manifold, since $d \eta \neq 0$, then we have

$$
\rho=0,
$$

which implies

$$
d f=0, \text { that is } f=\text { constant, }
$$

by virtue of (4.7). Thus using constancy of $f$ in (4.4), we obtain

$$
\stackrel{\star}{\operatorname{Ric}}(U, V)=-\rho g(U, V),
$$

for any $U, V \in T M$.

Hence we have the following:

Theorem 4.1. Let $M$ be a non-cosymplectic quasi-Sasakian 3-manifold. If $M$ admits a Ricci soliton $(v, \rho, g)$ with respect to the Schouten-van Kampen connection and $v$ is pointwise collinear with the structure vector field $\xi$, then $v$ is a constant multiple of the structure vector field, $M$ is an Einstein manifold with respect to the Schouten-van Kampen connection and the Ricci soliton is steady. 
Let us assume the converse, that is, let $M$ be an Einstein quasi-Sasakian 3-manifoldwith respect to the Schouten-van Kampen connection and $v=\xi$. Then we can write

$$
\stackrel{\star}{\operatorname{Ric}(U, V)}=\sigma g(U, V),
$$

where $\sigma$ is a scalar and $U, V \in T M$. From (2.3) and (4.1) we have $\left(\stackrel{\star}{L}_{\xi} g\right)(U, V)=0$, which implies that

$$
\left(\stackrel{\star}{L}_{\xi} g\right)(U, V)+2 \stackrel{\star}{R i c}(U, V)+2 \rho g(U, V)=2(\sigma+\rho) g(U, V) .
$$

From the previous equation it is obvious that $M$ admits a Ricci soliton $(\xi, \rho, g)$ if

$$
\sigma+\rho=0 .
$$

Thus we get the following:

Theorem 4.2. Let $M$ be a non-cosymplectic quasi-Sasakian 3-manifold. If $M$ is an Einstein manifold with equation $\stackrel{\star}{\text { Ric }}=\sigma g$, then the manifold admits a Ricci soliton $(\xi,-\sigma, g)$ with respect to the Schouten-van Kampen connection.

Now using (3.6) in (4.9), we get

$$
\operatorname{Ric}(U, V)+(\varphi U)[\beta] \eta(V)-2 \beta^{2} \eta(U) \eta(V)+\rho g(U, V)=0 .
$$

Using (2.10) in (4.12), we obtain

$$
\left(\frac{s c a l}{2}-\beta^{2}+\rho\right) g(U, V)+\left(\beta^{2}-\frac{s c a l}{2}\right) \eta(U) \eta(V)-(\varphi V)[\beta] \eta(U)=0 .
$$

Taking $U=\xi$ in (4.13), since $\rho=0$, we get $(\varphi V)[\beta]=0$. Now putting $V=\varphi V$, then we have

$$
V[\beta]=0,
$$

that is $\beta$ is a constant. Then we state the following:

Theorem 4.3. A quasi-Sasakian 3-manifold bearing a Ricci soliton $(\xi, \rho, g)$ with respect to the Schouten-van Kampen connection is a $\beta$-Sasakian manifold.

From (3.7), we also state the following:

Theorem 4.4. A quasi-Sasakian 3-manifold bearing a Ricci soliton $(\xi, \rho, g)$ with respect to the Schouten-van Kampen connection is of constant scalar curvature scal $=2 \beta^{2}$.

Now we consider $\eta$-Ricci soliton on a quasi-Sasakian 3-manifold with respect to the Schouten-van Kampen connection. Then we have

$$
\left.\stackrel{\star}{L}_{v} g+2 \stackrel{\star}{\star} i c+2 \rho g+2 \sigma \eta \otimes \eta\right)(U, V)=0,
$$

that is

$$
g\left(\nabla_{U} v, V\right)+g\left(U, \nabla_{V} v\right)+2 \stackrel{\star R i c}{ }(U, V)+2 \rho g(U, V)+2 \sigma \eta(U) \eta(V)=0 .
$$

Putting $v=\xi$ in (4.14), we obtain

$$
\stackrel{\star}{\operatorname{Ric}}(U, V)=-\rho g(U, V)-\sigma \eta(U) \eta(V) .
$$

Hence we state the following:

Theorem 4.5. A quasi-Sasakian 3-manifold bearing an $\eta$-Ricci soliton is an $\eta$-Einstein manifold with respect to the Schouten-van Kampen connection. 
Taking $V=\xi$ in (4.15), we get $\rho+\sigma=0$. Also using (3.6) in (4.15), we have

$$
\operatorname{Ric}(U, V)+(\varphi U)[\beta] \eta(V)-2 \beta^{2} \eta(U) \eta(V)+\rho g(U, V)+\sigma \eta(U) \eta(V)=0 .
$$

Using (2.10) in (4.16), we obtain

$$
\left(\frac{s c a l}{2}-\beta^{2}+\rho\right) g(U, V)+\left(\beta^{2}-\frac{s c a l}{2}+\sigma\right) \eta(U) \eta(V)-(\varphi V)[\beta] \eta(U)=0 .
$$

Taking $U=\xi$ in (4.17), since $\rho+\sigma=0$, we get $(\varphi V)[\beta]=0$. Now replacing $V$ by $\varphi V$ and using $\xi[\beta]=0$ gives

$$
V[\beta]=0 .
$$

Thefore we can state:

Theorem 4.6. A quasi-Sasakian 3-manifold bearing an $\eta$-Ricci soliton $(\xi, \rho, \sigma, g)$ with respect to the Schouten-van Kampen connection is a $\beta$-Sasakian manifold with $\rho+\sigma=0$.

If the vector field $v$ is the gradient of a potential function $-k$, then $g$ is called a gradient Ricci soliton that is $v=-\operatorname{grad} k$. In this case equation (1.3) becomes

$$
\nabla \operatorname{grad} k=R i c+\rho g,
$$

where $\nabla$ is the Levi-Civita connection. Now assume that $M$ is a quasi-Sasakian 3-manifold bearing a gradient Ricci soliton with respect to the Schouten-van Kampen connection. If we take $v=-\operatorname{grad} k$ in (4.1), we write

$$
\left(\stackrel{\star}{L}_{\text {grad } k} g\right)(U, V)=\left(L_{\text {grad } k} g\right)(U, V)=g\left(\nabla_{U} \operatorname{grad} k, V\right)+g\left(U, \nabla_{V} \operatorname{grad} k\right) .
$$

We can easily see that

$$
g\left(\nabla_{U} \operatorname{grad} k, V\right)=g\left(U, \nabla_{V} \operatorname{grad} k\right)
$$

which implies that

$$
\stackrel{\star}{L}_{\text {grad } k} g+2 \stackrel{\star}{R i c}+2 \rho g=0
$$

is equal to

$$
g\left(\nabla_{U} \operatorname{grad} k, V\right) \stackrel{\star}{\operatorname{Ric}}(U, V)+\rho g(U, V)
$$

This reduces to

$$
\nabla_{U} \operatorname{grad} k=\stackrel{\star}{Q} U+\rho U
$$

Now we will compute $R(U, V) \operatorname{grad} k=\left(\nabla_{U} Q\right) V-\left(\nabla_{V} Q\right) U$. Then we get

$$
\begin{aligned}
\nabla_{U} Q V= & \nabla_{U}\left\{\left(\frac{s c a l}{2}-\beta^{2}\right) U+\left(3 \beta^{2}-\frac{s c a l}{2}\right) \eta(U) \xi+\eta(U)(\varphi \operatorname{grad} \beta)-\varphi U[\beta] \xi\right\} \\
= & \left(\frac{1}{2} U[\text { scal }]-2 \beta U[\beta]\right) V+\left(6 \beta U[\beta]-\frac{1}{2} U[\operatorname{scal}]\right) \eta(V) \xi \\
& +\left(\frac{s c a l}{2}-\beta^{2}\right) \nabla_{U} V+\left(3 \beta^{2}-\frac{s c a l}{2}\right)\left(\left(\eta\left(\nabla_{U} V\right)+g\left(V, \nabla_{U} \xi\right)\right) \xi+\eta(V) \nabla_{U} \xi\right) \\
& +\left(\eta\left(\nabla_{U} V\right)+g\left(V, \nabla_{U} \xi\right)\right) \varphi \operatorname{grad} \beta+\eta(V) \nabla_{U} \varphi \operatorname{grad} \beta \\
& -U((\varphi V)[\beta]) \xi-\varphi V[\beta] \nabla_{U} \xi
\end{aligned}
$$

and

$$
\begin{aligned}
Q \nabla_{U} V= & \left(\frac{s c a l}{2}-\beta^{2}\right) \nabla_{U} V+\left(3 \beta^{2}-\frac{s c a l}{2}\right) \eta\left(\nabla_{U} V\right) \xi \\
& +\eta\left(\nabla_{U} V\right) \varphi \operatorname{grad} \beta-d \beta\left(\varphi \nabla_{U} V\right) \xi
\end{aligned}
$$


Then we have

$$
\begin{aligned}
\left(\nabla_{U} Q\right) V= & \nabla_{U} Q V-Q \nabla_{U} V \\
= & \left(\frac{1}{2} U[\text { scal }]-2 \beta U[\beta]\right) V+\left(6 \beta U[\beta]-\frac{1}{2} U[\text { scal }]\right) \eta(V) \xi \\
& +\left(3 \beta^{2}-\frac{s c a l}{2}\right)\left(g\left(V, \nabla_{U} \xi\right) \xi+\eta(V) \nabla_{U} \xi\right) \\
& +\left(\eta\left(\nabla_{U} V\right)+g\left(V, \nabla_{U} \xi\right)\right) \varphi \operatorname{grad} \beta+\eta(V) \nabla_{U} \varphi \operatorname{grad} \beta \\
& -U((\varphi V)[\beta]) \xi-\varphi V[\beta] \nabla_{U} \xi \\
& -\eta\left(\nabla_{U} V\right) \varphi \operatorname{grad} \beta+d \beta\left(\varphi \nabla_{U} V\right) \xi .
\end{aligned}
$$

which is equal to

$$
\begin{aligned}
\left(\nabla_{U} Q\right) V= & \nabla_{U} Q V-Q \nabla_{U} V \\
= & \left(\frac{1}{2} U[\text { scal }]-2 \beta U[\beta]\right) V+\left(6 \beta U[\beta]-\frac{1}{2} U[\text { scal }]\right) \eta(V) \xi \\
& -\beta\left(3 \beta^{2}-\frac{s c a l}{2}\right)(g(V, \varphi U) \xi+\eta(V) \varphi U) \\
& -\beta g(V, \varphi U) \varphi \operatorname{grad} \beta+\eta(V) \nabla_{U} \varphi \operatorname{grad} \beta \\
& -g\left(\nabla_{U} \operatorname{grad} \beta, \varphi V\right) \xi+\beta(\varphi V)[\beta] \varphi U-d \beta\left(\left(\nabla_{U} \varphi\right) V\right) \xi
\end{aligned}
$$

Putting $U=\xi$ in (4.18), we have

$$
\left(\nabla_{\xi} Q\right) V=\frac{1}{2} \xi[s c a l](V-\eta(V) \xi)+\eta(V) \nabla_{\xi} \varphi \operatorname{grad} \beta-g\left(\nabla_{\xi} \operatorname{grad} \beta, \varphi V\right) \xi-d \beta\left(\left(\nabla_{\xi} \varphi\right) V\right) \xi .
$$

Similarly, if we take $V=\xi$ in (4.18), we also have

$$
\left(\nabla_{U} Q\right) \xi=3 \beta U(\beta) \xi-\beta\left(3 \beta^{2}-\frac{s c a l}{2}\right) \varphi U+\nabla_{U} \varphi \operatorname{grad} \beta .
$$

Using (4.19) and (4.20), we obtain

$$
g\left(\left(\nabla_{U} Q\right) \xi-\left(\nabla_{\xi} Q\right) U, \xi\right)=3 \beta U(\beta)+g\left(\nabla_{\xi} \operatorname{grad} \beta, \varphi U\right)+d \beta\left(\left(\nabla_{\xi} \varphi\right) U\right) .
$$

Thus we can write

$$
g(R(\xi, U) \operatorname{grad} k, \xi)=3 \beta U(\beta)+g\left(\nabla_{\xi} \operatorname{grad} \beta, \varphi U\right)+d \beta\left(\left(\nabla_{\xi} \varphi\right) U\right) .
$$

Now we have

$$
R(U, \xi) \operatorname{grad} k=-U[\beta] \varphi(\operatorname{grad} k)-\beta^{2}\{g(U, \operatorname{grad} k) \xi-\eta(\operatorname{grad} k) U\}
$$

which implies that

$$
g(R(\xi, U) \operatorname{grad} k, \xi)=\beta^{2}\{g(U, \operatorname{grad} k)-g(\xi, \operatorname{grad} k) \eta(U)\} .
$$

From (4.21) and (4.22), we obtain

$$
3 \beta U[\beta]+g\left(\nabla_{\xi} \operatorname{grad} \beta, \varphi U\right)+d \beta\left(\left(\nabla_{\xi} \varphi\right) U\right)=\beta^{2}\{g(U, \operatorname{grad} k)-g(\xi, \operatorname{grad} k) \eta(U)\} .
$$

If $f$ is a constant, then we have $\operatorname{grad} k=\xi[k] \xi$. Using $g\left(\nabla_{V} \operatorname{grad} k, U\right)=\stackrel{\star}{\operatorname{Ric}}(U, V)+\rho g(U, V)$, we get

$$
\begin{aligned}
& \stackrel{\star}{\operatorname{Ric}}(U, V)+\rho g(U, V)=g\left(\nabla_{V} \xi(k) \xi, U\right) \\
& =V(\xi k) \eta(U)+(\xi k) g(U, V)-(\xi k) \eta(U) \eta(V) .
\end{aligned}
$$

Putting $U=\xi$ in (4.24), we obtain

$$
V(\xi k)=\rho \eta(V) .
$$

Now using (4.25) in (4.24), we get

$$
\stackrel{\star}{\operatorname{Ric}}(U, V)=(-\rho+(\xi k)) g(U, V)+(\rho-(\xi k)) \eta(V) \eta(U) .
$$

Hence we give the following: 
Theorem 4.7. A quasi-Sasakian 3-manifold bearing a gradient Ricci soliton with respect to the Schouten-van Kampen connection is an $\eta$-Einstein manifold provided $\beta$ is a constant.

In the last of this section, we consider a non-cosymplectic 3-dimensional quasi-Sasakian manifold $M$ bearing a Yamabe soliton defined by (1.4). Let $v$ be a pointwise collinear vector field with the structure vector field $\xi$, that is $v=f \xi$, where $f$ is a function on $M$. From (1.1) and (4.1) we write

$$
g\left(\nabla_{U} f \xi, V\right)+g\left(U, \nabla_{V} f \xi\right)=2(\stackrel{\star}{\star} c a l-\delta) g(U, V) .
$$

Using (2.3) in (4.26), we have

$$
(U f) \eta(V)+(V f) \eta(U)=2(\stackrel{\star}{\star} a l-\delta) g(U, V),
$$

by virtue of (3.6). By putting $V=\xi$ in (4.27), we obtain

$$
U f+(\xi f) \eta(U)=2(\stackrel{\star}{\star s} a l-\delta) \eta(U) .
$$

Taking $U=\xi$ in (4.28), we have

$$
\xi f=\stackrel{\star}{\star} a a l-\delta .
$$

If we replace (4.29) in (4.28), we get

$$
U f=(\stackrel{\star}{s c a l}-\delta) \eta(U),
$$

which yields

$$
d f=(\stackrel{\star}{\star} a l-\delta) \eta
$$

Applying $d$ on both sides of the last equation, we have

$$
\stackrel{\star}{\star} \text { scal }-\delta) d \eta=0 .
$$

In a non-cosymplectic quasi-Sasakian 3-manifold, since $d \eta \neq 0$ then we have

$$
\delta=\stackrel{\star}{\star} c^{\star} a l,
$$

which implies

$$
d f=0, \text { that is } f=\text { constant, }
$$

by virtue of (4.7).

Hence we state the followings:

Theorem 4.8. Let $M$ be a quasi-Sasakian 3-manifold bearing a Yamabe soliton $(v, \delta, g)$ with respect to the Schouten-van Kampen connection. If $v$ is pointwise collinear with the structure vector field $\xi$, then $v$ is a constant multiple of $\xi$ and $M$ is of constant scalar curvature with respect to the Schouten-van Kampen connection.

Corollary 4.1. A $\beta$-Sasakian 3-manifold bearing a Yamabe soliton $(\xi, \sigma, g)$ with respect to the Schouten-van Kampen connection is a manifold of constant scalar curvature with respect to the Levi-Civita connection.

\section{Examples}

In this section, we reconstruct examples of quasi-Sasakian 3-manifolds with respect to the Schouten-van Kampen connection.

Example 5.1. We consider the 3-manifold $M=\left\{(x, y, z) \in \mathbb{R}^{3},(x, y, z) \neq 0\right\}$ where $(x, y, z)$ are standard coordinate of $\mathbb{R}^{3}$. The vector fields

$$
e_{1}=\frac{\partial}{\partial x}-y \frac{\partial}{\partial z}, \quad e_{2}=\frac{\partial}{\partial y}, \quad e_{3}=\frac{\partial}{\partial z}
$$


are linearly independent at each point of $M$. Let $g$ be the Riemannian metric defined by

$$
\begin{aligned}
& g\left(e_{1}, e_{1}\right)=g\left(e_{2}, e_{2}\right)=g\left(e_{3}, e_{3}\right)=1, \\
& g\left(e_{1}, e_{2}\right)=g\left(e_{1}, e_{3}\right)=g\left(e_{2}, e_{3}\right)=0 .
\end{aligned}
$$

Assume that $\eta$ is a 1-form given by $\eta(Z)=g\left(Z, X_{3}\right)$, for any $Z \in \chi(M)$, and $\varphi$ is a $(1,1)$ tensor field defined by

$$
\varphi\left(e_{1}\right)=-e_{2}, \quad \varphi\left(e_{2}\right)=e_{1}, \quad \varphi\left(e_{3}\right)=0 .
$$

Then one can easily show that the quadruple $(\varphi, \xi, \eta, g)$ is an almost contact metric structure on $M$ by choosing $\xi=e_{3}$. Also, the Riemannian connection $\nabla$ of the metric $g$ is given by

$$
\begin{aligned}
2 g\left(\nabla_{X} Y, Z\right)= & X g(Y, Z)+Y g(X, Z)-Z g(X, Y) \\
& -g(X,[Y, Z])-g(Y,[X, Z])+g([X, Y], Z) .
\end{aligned}
$$

By direct calculations, we see that the non-zero components of the $\nabla$ on $M$ are

$$
\begin{aligned}
& \nabla_{e_{1}} e_{3}=-\frac{1}{2} e_{2}, \quad \nabla_{e_{1}} e_{2}=\frac{1}{2} e_{3}, \quad \nabla_{e_{2}} e_{3}=\frac{1}{2} e_{1}, \\
& \nabla_{e_{2}} e_{1}=-\frac{1}{2} e_{3}, \quad \nabla_{e_{3}} e_{2}=\frac{1}{2} e_{1}, \quad \nabla_{e_{3}} e_{1}=-\frac{1}{2} e_{2} .
\end{aligned}
$$

We see that the structure $(\varphi, \xi, \eta, g)$ satisfies the formula $\nabla_{X} \xi=-\beta \varphi X$ for $\beta=-\frac{1}{2}$. Hence the manifold is a quasi-Sasakian 3-manifold with the constant structure function $\beta$ [11]. Now with help of (3.1), we get the non-zero components of the $\stackrel{\star}{\nabla}$ on $M$ are

$$
\begin{aligned}
& \stackrel{\star}{\nabla}_{e_{1}} e_{3}=-\left(\frac{1}{2}+\beta\right) e_{2}, \quad \stackrel{\star}{\nabla_{e_{1}} e_{2}}=\left(\frac{1}{2}+\beta\right) e_{3}, \quad \stackrel{\star}{\nabla}_{e_{2}} e_{3}=\left(\frac{1}{2}+\beta\right) e_{1}, \\
& \stackrel{\star}{\nabla}_{e_{2}} e_{1}=-\left(\frac{1}{2}+\beta\right) e_{3}, \quad \stackrel{\star}{\nabla}_{e_{3}} e_{2}=\frac{1}{2} e_{1}, \quad \stackrel{\star}{\nabla}_{e_{3}} e_{1}=-\frac{1}{2} e_{2} .
\end{aligned}
$$

Using above equations, we can calculate the non-zero components of the curvature tensor $\stackrel{\star}{R}$ with respect to the Schouten-van Kampen connection

$$
\begin{array}{lll}
\stackrel{\star}{R}\left(e_{1}, e_{2}\right) e_{1} & =\frac{1}{2} e_{2}, & \stackrel{\star}{R}\left(e_{1}, e_{2}\right) e_{2}=-\frac{1}{2} e_{1}, \\
\stackrel{\star}{R}\left(e_{1}, e_{3}\right) e_{1}= & -\left(\frac{1}{4}+\frac{\beta}{2}\right) e_{3}, & \stackrel{\star}{R}\left(e_{1}, e_{3}\right) e_{2}=-\left(\frac{1}{4}+\frac{\beta}{2}\right) e_{3} .
\end{array}
$$

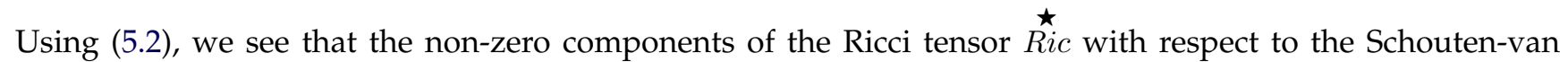
Kampen connection as follows:

$$
\stackrel{\star}{\operatorname{Ric}}\left(e_{1}, e_{1}\right)=\left(\frac{\beta}{2}-\frac{1}{4}\right), \quad \stackrel{\star}{R}\left(e_{2}, e_{2}\right)=\left(\frac{\beta}{2}-\frac{1}{4}\right) .
$$

For any $X, Y \in \chi(M)$, we write

$$
\begin{aligned}
& X=a_{1} e_{1}+a_{2} e_{2}+a_{3} e_{3} \\
& Y=b_{1} e_{1}+b_{2} e_{2}+b_{3} e_{3} .
\end{aligned}
$$

Then from (5.3), we have

$$
\begin{aligned}
\left(L_{\xi} g\right)(X, Y)+2 \stackrel{\star R i c}{ }(X, Y)+2 \rho g(X, Y)+2 \sigma \eta(X) \eta(Y)= & \left(\frac{\beta}{2}-\frac{1}{4}+\rho\right) a_{1} b_{1} \\
& +\left(\frac{\beta}{2}-\frac{1}{4}+\rho\right) a_{2} b_{2} \\
& +(\rho+\sigma) a_{3} b_{3} .
\end{aligned}
$$

If $\rho=\frac{1}{4}-\frac{\beta}{2}$ and $\sigma=\frac{\beta}{2}-\frac{1}{4}$, then $M$ admits an $\eta$-Ricci soliton $(\xi, \rho, \sigma, g)$ with respect to the Schouten-van Kampen connection. 
Example 5.2. Let $P$ be an open finite interval. Choose contants $a, b, c, d$ such that

$$
b \neq 0, y>a, d+2 c y>y^{2},
$$

for any $y \in P$. Let

$$
M=\mathbb{R} \times P \times \Re \subset \mathbb{R}^{3},
$$

and suppose that $(x, y, z)$ are coordinates on $M$ adapted from the Cartesian coordinates of $\mathbb{R}^{3}$. Define $g$ to be the Riemannian metric on $M$ given by

$$
\begin{aligned}
g= & (d+2 c y) d x \otimes d x+y(d x \otimes d z+d z \otimes d x) \\
& +b^{-2}(y-a)^{-1}\left(d+2 c y-y^{2}\right)^{-1} d y \otimes d y+d z \otimes d z .
\end{aligned}
$$

Let $\left\{e_{1}, e_{2}, e_{3}\right\}$ be linearly independent global frame on $M$ given by

$$
e_{1}=\left(d+2 c y-y^{2}\right)^{-1 / 2}\left(\frac{\partial}{\partial x}-y \frac{\partial}{\partial z}\right), \quad e_{2}=b(y-a)^{1 / 2}\left(d+2 c y-y^{2}\right)^{1 / 2} \frac{\partial}{\partial y}, \quad e_{3}=\frac{\partial}{\partial z} .
$$

The Poisson brackets of these vector fields are of the form

$$
\left[e_{1}, e_{2}\right]=-\alpha e_{1}+2 \beta e_{3}, \quad\left[e_{1}, e_{3}\right]=0, \quad\left[e_{2}, e_{3}\right]=0,
$$

where $\alpha, \beta$ are the functions on $M$ given by

$$
\begin{aligned}
\alpha & =b(y-c)(y-a)^{1 / 2}\left(d+2 c y-y^{2}\right)^{-1 / 2}, \\
\beta & =\frac{b}{2}(y-a)^{1 / 2} .
\end{aligned}
$$

Let $\eta$ be the 1-form defined by

$$
\eta(X)=g\left(X, e_{3}\right)
$$

for any $U \in T M$ and $\varphi$ be the $(1,1)$ tensor field defined by

$$
\varphi e_{1}=e_{2}, \quad \varphi e_{2}=-e_{1}, \quad \varphi e_{3}=0 .
$$

Then using the linearity of $\varphi$ and $g$, we have

$$
\eta\left(e_{3}\right)=1, \quad \varphi^{2} U=-U+\eta(U) e_{3}
$$

and

$$
g(\varphi X, \varphi Y)=g(X, Y)-\eta(X) \eta(Y), \quad X, Y \in T M .
$$

Using the above formula for Riemannian metric $g$, we can show that the non-zero components of the $\nabla$ on $M$ are

$$
\begin{aligned}
& \nabla_{e_{1}} e_{3}=-\beta e_{2}, \quad \nabla_{e_{2}} e_{3}=\beta e_{1}, \\
& \nabla_{e_{3}} e_{1}=-\beta e_{2}, \quad \nabla_{e_{1}} e_{2}=-\alpha e_{1}+\beta e_{3}, \quad \nabla_{e_{2}} e_{1}=-\beta e_{3}, \\
& \nabla_{e_{3}} e_{2}=\beta e_{1}, \quad \nabla_{e_{1}} e_{1}=\alpha e_{2} .
\end{aligned}
$$

Hence from (2.3), the manifold $M$ is a 3-dimensional quasi-Sasakian manifold with $\beta=1$ [24]. With help of (3.1), we get the non-zero components of the $\stackrel{\star}{\nabla}$ on $M$ are

$$
\begin{aligned}
& \stackrel{\star}{\nabla}_{e_{3} e_{2}}=\beta e_{1}, \quad \stackrel{\star}{\nabla}_{e_{1}} e_{1}=\alpha e_{2}, \\
& \stackrel{\star}{\nabla}_{e_{3}} e_{1}=-\beta e_{2}, \quad \stackrel{\star}{\nabla}_{e_{1}} e_{2}=-\alpha e_{1},
\end{aligned}
$$

Using (5.8), we can calculate the non-zero components of its curvature tensor with respect to the Schouten-van Kampen connection as follows:

$$
\stackrel{\star}{R}\left(e_{1}, e_{2}\right) e_{1}=\left(\alpha^{2}+2 \beta^{2}\right) e_{2}, \quad \stackrel{\star}{R}\left(e_{1}, e_{2}\right) e_{2}=-\left(\alpha^{2}+2 \beta^{2}\right) e_{1},
$$


From (5.9), we see that the non-zero components of the Ricci tensor with respect to the Schouten-van Kampen connection as follows:

$$
\stackrel{\star}{\operatorname{Ric}}\left(e_{1}, e_{1}\right)=-\left(\alpha^{2}+2 \beta^{2}\right), \quad \stackrel{\star}{\operatorname{Ric}}\left(e_{2}, e_{2}\right)=-\left(\alpha^{2}+2 \beta^{2}\right) .
$$

For any $X, Y \in \chi(M)$, we write

$$
\begin{aligned}
& X=a_{1} e_{1}+a_{2} e_{2}+a_{3} e_{3} \\
& Y=b_{1} e_{1}+b_{2} e_{2}+b_{3} e_{3} .
\end{aligned}
$$

Then we have

$$
\begin{aligned}
\left(L_{\xi} g\right)(X, Y)+2 \stackrel{\star}{\operatorname{Ric}}(X, Y)+2 \rho g(X, Y)+2 \sigma \eta(X) \eta(Y)= & -\left(\alpha^{2}+2 \beta^{2}+\rho\right) a_{1} b_{1} \\
& +\left(-\alpha^{2}-2 \beta^{2}-\rho\right) a_{2} b_{2} \\
& +(\rho+\sigma) a_{3} b_{3} .
\end{aligned}
$$

If $\rho=-\alpha^{2}-2 \beta^{2}$ and $\sigma=\alpha^{2}+2 \beta^{2}$, then $M$ admits an $\eta$-Ricci soliton $(\xi, \rho, \sigma, g)$ with respect to the Schouten-van Kampen connection.

\section{References}

[1] Barbosa E., Riberio E.: On conformal solutions of the Yamabe flow. Arch. Math. 101, 79-89 (2013).

[2] Blair D. E.: Contact manifolds in Riemannian geometry. Lecture Notes in Mathematics Vol 509. Springer-Verlag, Berlin-New York (1976).

[3] Blair D. E.: Riemannian geometry of contact and symplectic manifolds. Progress in Mathematics Vol 203. Birkhauser Boston Inc. (2002).

[4] Blair D. E.: The theory of quasi-Sasakian structure. J. Differential Geo. 1, 331-345 (1967).

[5] Bejancu A., Faran H.: Foliations and geometric structures. Math. and Its Appl. 580. Springer, Dordrecht (2006).

[6] Cao H. D., X. Sun X., Zhang Y.: On the structure of gradient Yamabe solitons. Mathematical Research Letters. 19, 767-774 (2012).

[7] Chen B. Y., Deshmukh S.: Yamabe and quasi Yamabe solitons on Euclidean submanifolds. Mediterr. J. Math. 15(5), 1-9 (2018).

[8] Cho J. C., Kimura M.: Ricci solitons and real hypersurfaces in a complex space form. Tohoku Math. J. 61(2), 205-212 (2009).

[9] Chow B., Knopf D.: The Ricci flow: An introduction, Mathematical Surveys and Monographs 110. American Math. Soc. (2004).

[10] De U. C., Yildiz A., Sarkar A.: Isometric immersion of three-dimensional quasi-Sasakian manifolds. Math. Balkanica. 22(3-4), 297-306 (2008).

[11] De U. C., Mandal A. K.: 3-dimensional quasi-Sasakian manifolds and Ricci solitons, Sut Journal of Mathematics. 48(1), 71-81 (2012).

[12] Derdzinski A.: Compact Ricci solitons. preprint.

[13] Deshmukh S., Chen B. Y.: A note on Yamabe solitons. Balkan J. Geom. Appl. 23(1), 37-43 (2018).

[14] Gonzalez J. C. , Chinea D.: Quasi-Sasakian homogeneous structures on the generalized Heisenberg group H(p,1). Proc. Amer. Math. 105, 173-184 (1989).

[15] Hamilton R. S.: The Ricci flow on surfaces. Mathematics and general relativity, Contemp. Math. 71, 237-262 (1988).

[16] Ianuş S.: Some almost product structures on manifolds with linear connection. Kodai Math. Sem. Rep. 23, 305-310 (1971).

[17] Ivey T.: Ricci solitons on compact 3-manifolds. Differential Geo. Appl. 3, 301-307 (1993).

[18] Janssens D., Vanhecke L.: Almost contact structures and curvature tensors. Kodai Math. J. 4(1), 1-27 (1981).

[19] Kim B. H.: Fibred Riemannian spaces with quasi-Sasakian structure. Hiroshima Math. J. 20, 477-513 (1990).

[20] Kanemaki S.: Quasi-Sasakian manifolds. Tohoku Math. J. 29, 227-233 (1977).

[21] Kanemaki S.: On quasi-Sasakian manifolds. Differential Geometry Banach Center Publications. 12, 95-125 (1984).

[22] Neto, B. L.: A note on (anti-)self dual quasi Yamabe gradient solitons. Results Math. 71, 527-533 (2017).

[23] Olszak Z.: Normal almost contact metric manifolds of dimension 3. Ann. Polon. Math. 47, 41-50 (1986).

[24] Olszak Z.: On three dimensional conformally flat quasi-Sasakian manifold. Period Math. Hungar. 33(2), 105-113 (1996).

[25] Olszak Z.: The Schouten-van Kampen affine connection adapted an almost (para) contact metric structure. Publ. De L'inst. Math. 94, 31-42 (2013).

[26] Oubina J. A.: New classes of almost contact metric structures. Publ. Math. Debrecen. 32, 187-193 (1985).

[27] Perelman G.: The entopy formula for the Ricci flow and its geometric applications, Preprint arxiv:0211159 (2002).

[28] Schouten J., van Kampen E.: Zur Einbettungs-und Krümmungsthorie nichtholonomer Gebilde. Math. Ann. 103, 752-783 (1930).

[29] Sharma R.: Certain results on K-contact and $(k, \sigma)$ - contact manifolds. Journal of Geometry. 89, 138-147 (2008).

[30] Solov'ev A. F.: On the curvature of the connection induced on a hyperdistribution in a Riemannian space. Geom. Sb. 19, 12-23 (1978).

[31] Solov'ev A. F.: The bending of hyperdistributions. Geom. Sb., 20(1979), 101-112, (in Russian).

[32] Solov'ev A. F.: Second fundamental form of a distribution. Mat. Zametki, 35, 139-146 (1982).

[33] Solov'ev A. F.: Curvature of a distribution. Mat. Zametki. 35, 111-124 (1984).

[34] Tanno S.: Quasi-Sasakian structure of rank 2p+1. J. Differential Geom. 5, 317-324 (1971).

[35] Yano K., Kon M.: Structure on manifolds. World Scientific (1984). 


\section{Affiliations}

SELCEN YÜKSEL PERKTAŞ

ADDRESS: Adıyaman University, Dept. of Mathematics, 02040, Adıyaman-Turkey.

E-MAIL: sperktas@adiyaman.edu.tr

ORCID ID:0000-0002-8848-0621

AHMET YILDIZ

AdDRESS: İnönü University, Dept. of Mathematics and Science Education, 44000, Malatya-Turkey. E-MAIL: a.yildiz@inonu.edu.tr

ORCID ID: 0000-0002-9799-1781 